\title{
Bilateral Accessory Middle Turbinate: A Rare Anatomical Varaition.
}

\author{
Dr. Rahul Kawatra ${ }^{1}$, Dr. Puneet Maheshwari ${ }^{2}$, Dr. Shikhar Saxena ${ }^{3}$, \\ Dr. Parsoon Varshney ${ }^{4}$ \\ ${ }^{\text {I }}$ Head of Department of otorhinolaryngology, Era's Lucknow Medical college and Hospital/Dr RML Avadh \\ University Faizabad, India) \\ ${ }^{2}$ (Senior Resident, Department of otorhinolaryngology, Era's Lucknow Medical college and Hospital/Dr RML \\ Avadh University Faizabad, India) \\ ${ }^{3}\left(J u n i o r\right.$ resident $2^{\text {nd }}$ year, Department of otorhinolaryngology, Era's Lucknow Medical college and Hospital/Dr \\ RML Avadh University Faizabad, India) \\ ${ }^{4}$ (Junior resident $1^{\text {st }}$ year, Department of otorhinolaryngology, Era's Lucknow Medical college and Hospital/Dr \\ RML Avadh University Faizabad, India)
}

\begin{abstract}
A bilateral accessory middle turbinate is an extremely rare anatomical variation. The embryological development of the nasal turbinate is a complex process during which anatomic variations may occur at multiple points. It is a bony prominence covered with soft tissue that originates from the lateral wall. In this study, we report a case of bilateral accessory middle turbinate projecting inferolaterally leading to chronic nasal obstruction. With the advent of imaging techniques like CT scans of paranasal sinuses, and diagnostic nasal endoscopy, the anatomical variations of nose and paranasal sinuses can easily be elicited. In this case after confirming the diagnosis by CT scan and diagnostic nasal endoscopy the patient was electively posted for Functional Endoscopic Sinus Surgery and bilateral accessory middle turbinates were resected out.
\end{abstract}

Keywords: Accessory middle turbinate, Functional Endoscopic Sinus Surgery, Osteomeatal complex.

\section{Introduction}

The permanent middle turbinate develops from the second ethmoturbinal. It is the most constant and complete lamella formation and separates the anterior and posterior ethmoidal labyrinths, thus forms an important landmark for surgery. Middle turbinate is associated with various functions including regulation of airflow and temperature, humidification, filtration, lubrication of upper airways and olfaction.[1] Anatomical variations are frequently encountered in nose and paranasal sinuses of which pneumatization of middle turbinate is commonest, this condition is known as concha bullosa.[2] Khanobthanchai et all were the first to describe secondary middle turbinate as a bony projection originating from lateral wall of middle meatus, covered by soft tissue.[3]Accessory middle turbinate is also another variation where uncinate process is medially bent and anteriorly folded, giving a appearance of middle turbinate.[4]We report a case of bilateral accessory middle turbinate with chronic nasal obstruction.

\section{Case report}

A 56 year old caucasian male, presented with history of on and off epistaxis, nasal obstruction and headache since two years. Patient had no complaints of rhinorrhea or any post nasal discharge. Patient had no history of any trauma or nasal surgery. Patient was not under any medical treatment. Anterior rhinoscopic examination revealed left sided deviated nasal septum with bilateral inferior turbinate hypertrophy. Diagnostic nasal endoscopy revealed enlarged uncinate bilaterally, mimicking bifid middle turbinate[Fig i]. CT scan of paranasal sinuses was performed which showed medial bending of uncinate process bilaterally,confirming the diagnosis of accessory middle turbinate[Fig.ii] .All the paranasal sinuses were clear. There was no concha bullosa. Other laboratory investigations were found within normal limits. The patient underwent transnasal endoscopic surgery under general anesthesia and bilateral uncinectomy was done and enlarged uncinte was resected out thus relieving the nasal obstruction [Fig iii].

Histopathological examination of specimen excluded the presence of any polyp, benign or malignant lesion. Post operatively patient was given saline nasal spray for one month under the cover of antibiotics and decongestants. Patient's symptoms diminished in the post operative period of one month. Post operatively nasal endoscopy revealed normal epithelization with no complications. 


\section{Discussion}

A series of outgrowths from lateral nasal wall lead to formation of nasal turbinates. The outgrowths form series of ridges, separated by furrows and are known as ethmoturbinals. [5,6,7] Six ridges develop initially, out of which some fuse together to form permanent turbinates. From ascending portion of first ridge develops agger nasi and from its descending portion develops the uncinate process. [6] The middle turbinate and superior turbinate develops from third and fourth ridge, thus forming the first and second permanent ethmoturbinals respectively. The middle turbinate plays a functional role in the nasal physiology, including lamination of airflow, humidification of inspired air and its deflection superiorly towards the olfactory epithelium. Secretions produced by the nasal and paranasal sinus mucosal lining are transported along the surface of middle turbinate to the nasopharynx. Thus surface and dimensions of middle turbinate are crucial for maintenance of normal nasal physiology.

Middle turbinate is used as an important reference point during endoscopic sinus surgery. Thus the knowledge of anatomical anomalies of middle turbinate is important in evaluating patients with nasal and paranasal sinus diseases. The common anatomical variations of middle turbinate are Concha bullosa, Secondary middle turbinate, Accessory middle turbinate.

a)The most common variation is pneumatization of the turbinates, especially the concha bullosa of the middle turbinate.It is reported to range between 14 and $80 \%$.Treatment is endoscopic conchactomy, if symptomatic.

b) Khanobthamchai et al [3] were the first to describe that secondary middle turbinate, was an incomplete anterior wall of bulla ethmoidalis. Some suggested that it could originate from frontal ridge which normally forms anterior ethmoidal cells. The secondary middle turbinate is generally located posterosuperior to infundibulum and then curves medially and superiorly.

c) Accessory middle turbinate is a very rare anomaly described as medially bent and inferiorly folded uncinate process understandably, the patient with Accessory middle turbinate has no uncinate process.

To diagnose these anatomical variations the CT scan paranasal sinus along with diagnostic nasal endoscopy are the key investigative procedures. The treatment should be carried out only if the patient is symptomatic. The recommended treatment is Functional Endoscopic Sinus Surgery to resect the anatomical variation and to establish the patency of osteomeatal complex. Post operatively patient should be prescribed with topical decongestants -, antibiotics and saline nasal spray to decongest the mucosa , prevent any infection and to prevent synachia formation respectively.

\section{Conclusion}

Accessory middle turbinate is an uncommon anatomical variation of middle turbinate. This variation may have no clinical consequence. CT scans can easily identify such uncommon anatomical variations. However in symptomatic patients, if functional sinus surgery is contemplated, it is important to be aware of these variations to avoid any complications during surgery.

\section{References}

[1] Lee HY, Kim CH, Kim JY, et al, Surgical anatomy of idle turbinate, ClinAnat ,19, 2006, 493-6.

[2] Bolger WE, Butzin CA, and Parsons DS,Paranasal sinus bony anatomic variations and mucosal abnormalities : CT analysis for endoscopic sinus surgery. Laryngoscope, 101, 1991, 56-64.

[3] Khanobthamchai K, Shankar L, Hawke M, and Bingham B, The secondary middle turbinate, J Otolaryngol, 20, 1991, $412-413$.

[4] Lin YL, Lin YS, Su WF, and Wang CH, A secondary middle turbinate co existing with an accessory middle turbinate: an unusual combination of two anatomical variations, ActaOtolaryngol, 126, 2006, 429-431.

[5] Stammberger H, Functional endoscopic sinus surgery (Pheladelphia : B.C .Decker, 1991.)

[6] Stammberger H, Braun H. Pneumatization of turbinates, Laryngoscope, 113, 2003, 668-672.

[7] Lund V, Anatomy of nose and paranasal sinuses, In: Gleeson M, Kerr A G, edsscottbrown"s otolaryngology, Basic Sciences, $6^{\mathrm{TH}}$ ed, Oxford, Butterworth-Heinemann, 1997, 1/5/4. 
Figures

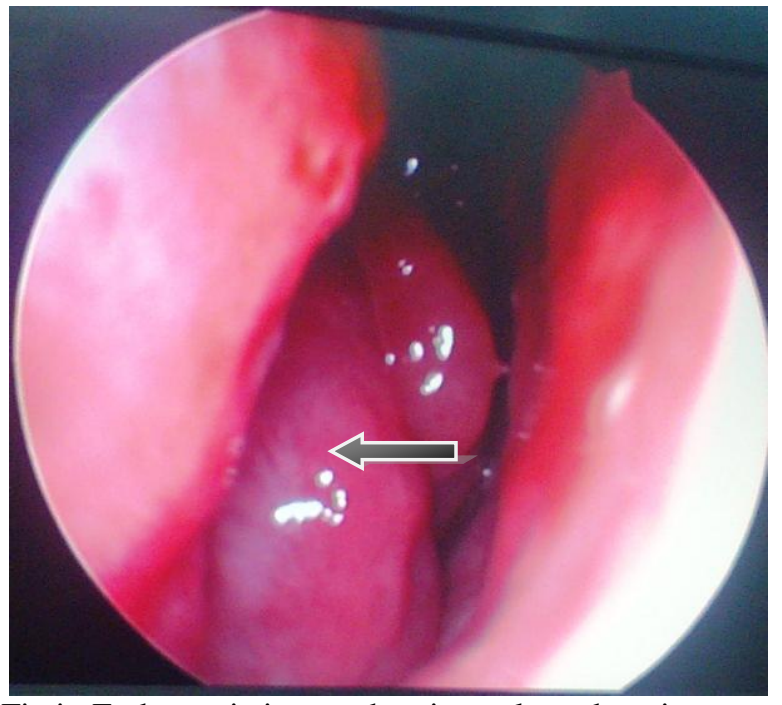

Fig i : Endoscopic image showing enlarged uncinate process, mimicking bifid middle turbinate- Accessory Middle Turbinate.

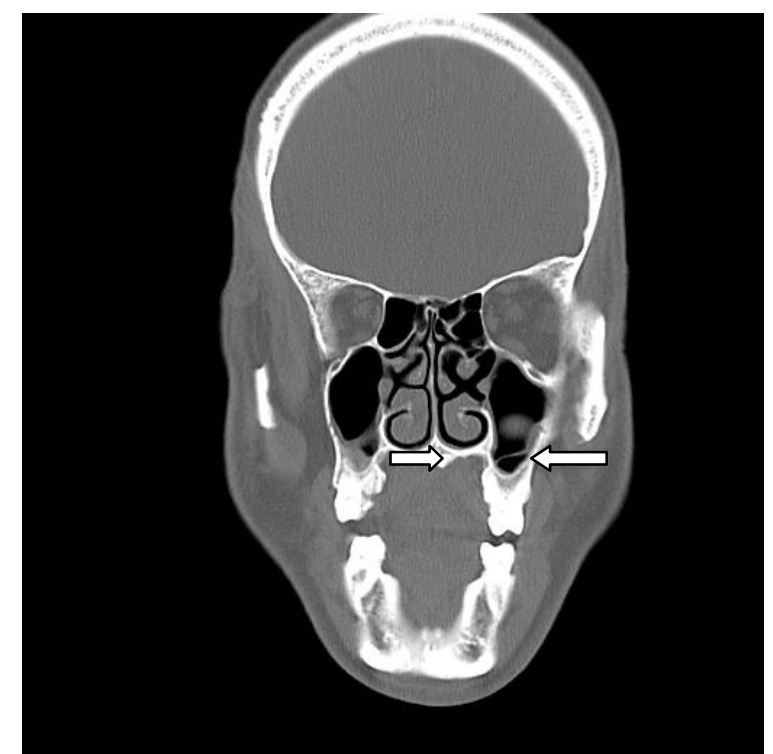

Fig ii : CT Scan Para nasal sinus, showing bilateral enlarged and medially bent uncinate. The Accessory Middle turbinate.

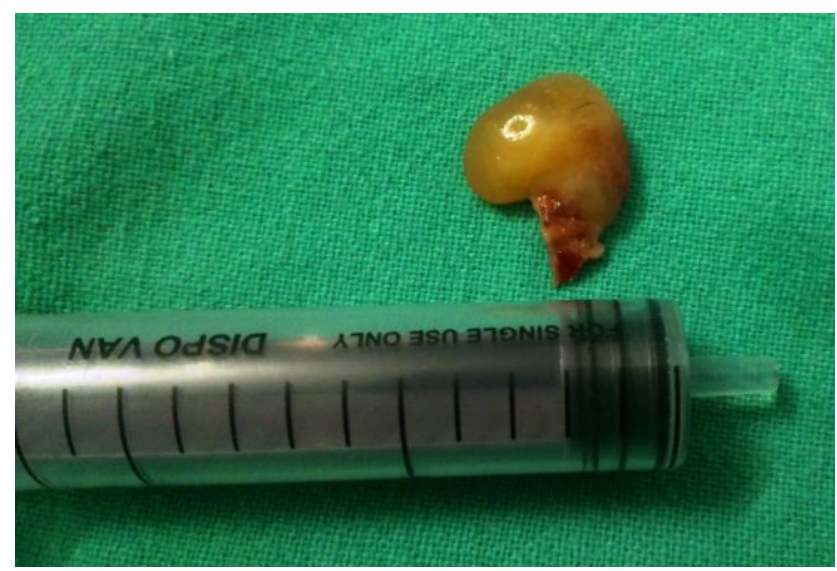

Fig iii : Endoscopically excised enlarged uncinate process. 